\title{
An automated CFD analysis workflow in overall aircraft design applications
}

\author{
Xiangyu Gu${ }^{1}$ ([) $\cdot$ Pier Davide Ciampa ${ }^{1} \cdot$ Björn Nagel $^{1}$
}

Received: 10 May 2016/Accepted: 19 September 2017/Published online: 28 October 2017

(c) The Author(s) 2017. This article is an open access publication

\begin{abstract}
An automated CFD-based analysis process for applications at the early aircraft development stages is presented. The robustness of the implemented process, which relies on a knowledge-based layer implemented into the automated pre-processing step of the geometrical components, allows taking advantage of high fidelity simulations, also for large explorations of the design space. The well-known aircraft configuration DLR-F6 is chosen to verify the automated analysis process. The CFD analysis process is integrated into the DLR multi-fidelity aircraft design environment, which relies on the DLR open source distributed framework RCE, and the DLR central data model CPACS. The overall aircraft design synthesis is performed for a conventional passenger transportation aircraft configuration, by making use of variable fidelity methods for the aerodynamic analysis. The results discuss the impact of employing CFD-based analysis into overall aircraft design applications.
\end{abstract}

Keywords CPACS - Automated mesh generation - CFD . Multi-fidelity aircraft design

$\begin{array}{ll}\text { Abbreviations } \\ b & \text { Wing span } \\ C_{\mathrm{L}} & \text { Lift coefficient }=\frac{\mathrm{Lift}}{\frac{1}{2} \rho \nu_{\infty}^{2} S_{\text {ref }}} \\ C_{\mathrm{D}} & \text { Drag coefficient }=\frac{\mathrm{Drag}}{\frac{1}{2} \rho \rho_{\infty}^{2} S_{\text {ref }}} \\ C_{\mathrm{P}} & \text { Pressure coefficient }=\frac{P-P_{\infty}}{\frac{1}{2} \rho v_{\infty}^{2}}\end{array}$

Xiangyu Gu

Xiangyu.gu@dlr.de

1 Institute of System Architectures in Aeronautics, Aircraft Design \& System Integration, German Aerospace Center, 21129, ZAL TechCenter, Hein-Saß-Weg 22, Hamburg, Germany
$C_{\text {ref }} \quad$ Wing reference chord (m)

$S_{\text {ref }} \quad$ Reference area $\left(\mathrm{m}^{2}\right)$

Ma Far field Mach number

$R e_{\mathrm{c}} \quad$ Reynolds number based on $C_{\text {ref }}$

AOA Angle of Attack (deg)

$H \quad$ Fraction of wing semi-span

TLAR Top level aircraft requirement

MTOW Maximum takeoff weight $(\mathrm{kg})$

OEM Operating empty mass (kg)

MFM Fuel mass (kg)

OAD Overall aircraft design

MDAO Multidisciplinary design analysis and optimization

\section{Introduction}

The increasing demand for commercial aviation and the growing environmental concerns have become the key drivers in improving aircraft fuel efficiency. The ICAO Programme of Action on International Aviation and Climate Change, which targets a $2 \%$ improvement in global fuel efficiency annually until the year 2050 [1], and the ACARE Strategic Research and Innovation Agenda (SRIA) [2], are examples of such ambitious targets.

Unconventional aircraft configurations, such as the Blended Wing Body [3] (BWB) and the strut-braced wing [4], are promising candidates to significantly improve the fuel efficiency. Typically, at the conceptual design stages of an aircraft development program, the Top Level Aircraft Requirements (TLAR), such as the transportation mission, operational constraints, etc., are specified and the overall aircraft synthesis is performed based on statistics or empirical design rules. However, unlike for conventional aircraft designs, novel configurations suffer from the lack 
of empirical knowledge. Due to the high development costs and the economic risks associated with unconventional configurations, from the beginning of the design phase, it is necessary to correctly predict the configuration's behavior, to guarantee the promised performance. On the other hand, the process of computer technology over last decades is pushing the application of physics-based disciplinary tools also in the aircraft conceptual design stage [5]. However, including physics-based analyses in the early stages requires the generation of the analysis input models in a time efficient manner. Furthermore, to assess the aircraft overall heterogeneous disciplinary analysis modules need to be integrated in the same design process. As soon as the interdisciplinary dependencies are accounted into the design process, the application of Multidisciplinary Design Analysis and Optimization (MDAO) techniques can support the designers to correctly capture the overall aircraft's behavior. Further, the shift to physics-based analysis at the beginning of the design cycle is associated with the increase of the "aircraft modeling complexities" [6] which typically leading to an increased number of the design variables, and a higher domain expertise required to set up the analysis parameters. Hence, in a design process which makes use of physics-based analysis module the designers' team faces the following challenges:

- Generation of an initial design, with a sufficient quality, and details, to serve the instantiation of further physicsbased analysis modules.

- Automate the setup of an increased number of parameters, and design variables, associated to execution of the physics-based analysis modules.

- Handle and setup consistent disciplinary couplings in MDAO applications, for a multitude of heterogeneous analysis tools.

The aforementioned challenges depend on the complexity of the modeling, and on the physics phenomena representation supported by the disciplinary analysis. Hence, the following disciplinary fidelity levels can be identified [7]:

- Level 0 consisting of typical conceptual Overall Aircraft Design (OAD) approaches, based on empirical relations, and existing databases.

- Level 1 refers to disciplinary analysis based on simplification on the modeling, and on the representation of the physics phenomena, mainly accounting for linear effects.

- Level 2 refers to an accurate modeling of the aircraft components, accounting for a higher level of details, and physics representation accounting for non-linear phenomena;

- Level 3 refers to the state of the art of physics simulations, mainly dedicated to non-linear local effects, and whose disciplinary models is hardly to be fully automated, as required for extensive MDAO applications.

The introduced fidelity classification and corresponding wall time estimates to perform a single point aerodynamic analysis are indicated in Table 1.

As most of the current large commercial aircraft operates in the transonic flight regime during the cruise phase, accurate wave drag assessment is essential for the design trade-off. Currently, the Vortex lattice method (VLM) is widely used to evaluate the aerodynamic performance in the aircraft early design stages. However, even if corrections can be applied, it is not capable to account for the wave drag at cruise condition. On the other hand, the improvement of computational efficiency, as well as the matureness of Computational Fluid Dynamics (CFD) techniques over the past decades, allows engineers to make use of CFD to accurately predict the flow field, even at the critical transonic conditions and within acceptable analysis time and affordable computational resources. Hence, it becomes necessary and possible to adopt CFD to evaluate the aerodynamic performance in early design stages.

CFD analysis requires an accurate and water-tight representation of the aircraft wetted surface, or Outer Mould Line (OML). Besides, the generation of CFD meshes, requires extensive, and usually manual pre-processing operations of the geometry components. Further, at the early design stages, these operations may be repeated multiple times to extensively explore the design space, and to investigate large geometry variations. Hence, the automation of geometrical pre-processing operations and of the mesh generation step constitutes the main challenges to employ CFD within overall aircraft design applications.

This paper presents an automated CFD-based analysis chain, aiming to improve the prediction of the aerodynamic behavior at cruise condition in the pre-design stages, and bringing CFD analysis into the overall aircraft synthesis process. In this study, the aircraft product representation is based on the DLR Common Parametric Aircraft Configuration Schema (CPACS) data modeling.

The paper is organized as follows. The implementation process is presented in Sect. 2. In this section the geometry

Table 1 Aerodynamic analysis fidelity level classification

\begin{tabular}{lll}
\hline Level & Aerodynamics & Wall time \\
\hline L0 & Empirical performance estimation & $<1 \mathrm{~s}$ \\
L1 & Subsonic simulation (VLM, Panel method) & Minutes \\
L2 & Transonic inviscid simulation (Euler) & Hours \\
L3 & Viscous simulation (RANS) & Days \\
\hline
\end{tabular}


Fig. 1 CFD analysis workflow in RCE

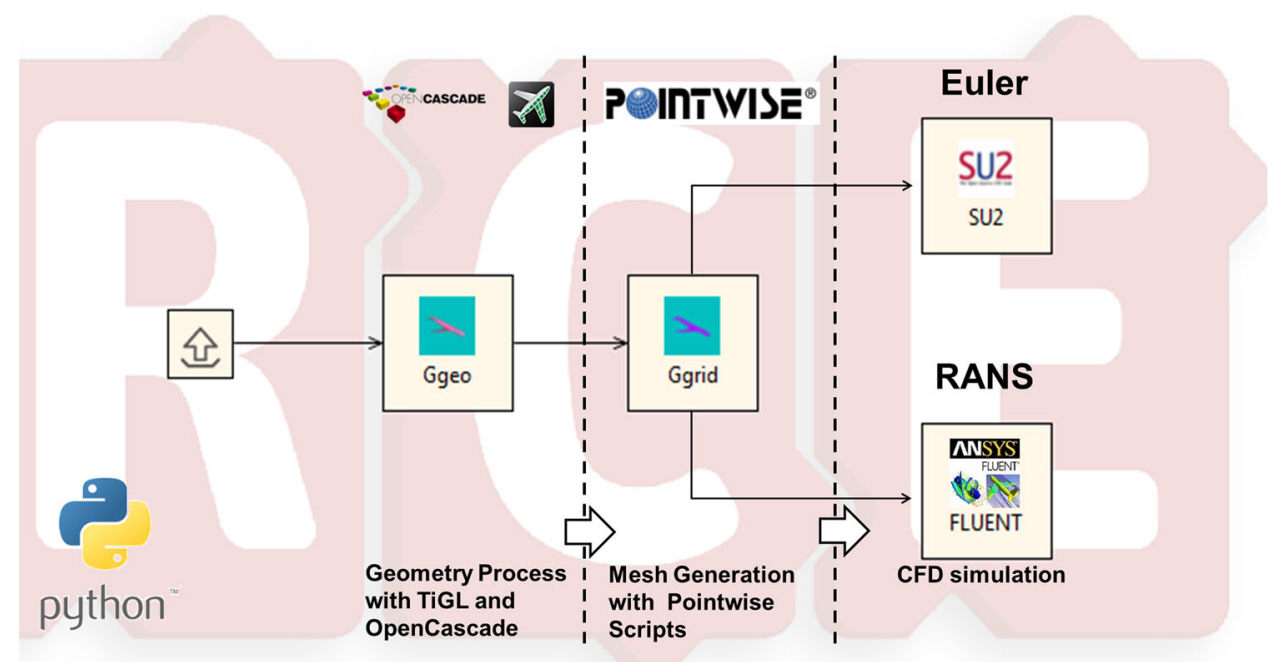

representation, the automated mesh generator and the CFD solver used in the analysis process are described. In Sect. 3, the analysis process is applied to the DLR-F6 wing body configuration to verify the geometry representation and the mesh generation. In Sect. 4, the analysis process is used within the aircraft synthesis of a passenger transportation aircraft. Mission analysis results, such as mission fuel, are compared against the synthesis results when employing only empirical-based method, and the ones when the aerodynamics characteristics rely on VLM-based methodologies. Conclusions and outlook of the article are provided in the last section.

\section{Automated CFD-based analysis process}

To foster the collaboration among disciplinary specialists and the integration of disciplinary expertise into the overall aircraft design process, the centralized data structure CPACS [8] has been developed by DLR over last decade. It contains information of the product model, such as its geometry description, and holds process data to control the overall analysis workflow. To support the handling of CPACS-described geometries to be progressed to the disciplinary analysis, the dedicated library TiGL [9] (TIVA Geometry Library) has being developed by DLR. The TiGL Geometry Library which is based on OpenCASCADE [10] kernel represents the airplane's components geometry by B-spline surfaces, and it can export the geometry as CAD-based format for further disciplinary analysis.

The analysis process in this study makes use of the CPACS model description to facilitate the integration of CFD-based analysis with the other discipline analysis within the DLR overall aircraft design framework. The analysis process is designed to fully automated to meet the aforementioned requirements of the early design stages. The overall process, starting with the processing of the geometrical CPACS description, to the results of the aerodynamic solution, has been implemented to be flexible and fully automated for arbitrary configuration input. The robustness of the developed process, which relies on the knowledge-based layer implemented into the pre-processing components, allows taking advantage of high fidelity simulations, also for large explorations of the design space, as typically required at the early development stages.

The engineering framework chosen for the implementation of the workflow process in this study is the open source integration distributed engineering environment RCE (Remote Component Environment), developed by DLR [11]. A representation of the implemented workflow is illustrated in Fig. 1, and the individual components are described in Table 2 and the following sub-sections.

\subsection{Geometry representation}

The first component of the implemented chain, named Ggeo, is responsible to translate the aircraft CPACS description, into a CAD-based model suitable for CFD mesh applications (L2, L3). With an arbitrary valid CPACS files as input, the component automatically generates a CAD models (iges, step, etc.) for each aircraft component that user is willing to include in the analysis, respectively. The component, which is based on the OpenCascade kernel accessible via API TiGL library, is implemented in Python. Ggeo makes use of the CPACS hierarchical structure to identify the aircraft geometry topology, such as the numbers of wings, fuselages and the connectivity information. Besides, the dimensional information of each aircraft component is also calculated by the TiGL functions. Then the CAD model and topology and dimensional information are passed to the following disciplinary analysis modules. 
Table 2 Components description of the CFD analysis process

\begin{tabular}{lllll}
\hline & Geometry generation & Mesh generation & Analysis & RANS \\
\cline { 3 - 5 } & & & Euler & FLUENT \\
$\begin{array}{l}\text { Component } \\
\text { Name }\end{array}$ & Ggeo & Ggrid & SU2 & Solve RANS equations, \\
$\begin{array}{l}\text { Function } \\
\begin{array}{c}\text { Software } \\
\text { dependencies }\end{array}\end{array}$ & $\begin{array}{c}\text { Generates a CAD object from } \\
\text { a valid CPACS file }\end{array}$ & $\begin{array}{c}\text { Generates a grid for Euler/RANS } \\
\text { analysis form a CAD file }\end{array}$ & $\begin{array}{c}\text { Solve Euler equations, } \\
\text { inviscid simulation }\end{array}$ & $\begin{array}{c}\text { Siscous simulation } \\
\text { Pointwise }\end{array}$ \\
\hline
\end{tabular}

\subsection{Knowledge-based mesh generator}

The mesh generation component, named Ggrid, is an under-development Python-based tool, which automatically generates macros for the mesh generators, to produce isotropic tetrahedral mesh for inviscid flow simulation, and hybrid or anisotropic tetrahedral meshes for viscous flow simulation. In this study, the exported macros are compatible with Pointwise [12] meshing tool.

The component can read the incoming CAD file and make Boolean manipulations to identify the intersections of components. According to the geometrical information incoming from the previous component, the macros will distribute default sources with the pre-implemented knowledge in the "critical positions", such as the leading edges, the trailing edges of the wings, and the intersections of wings and fuselages, shown in Fig. 2. Then, the macros will apply size functions according to the dimensional information coming from previous component with the pre-implemented knowledge to the boundary connectors.

In the component, a global factor $f$ is implemented to control the grid size settings according to the compromise of computational resources cost and the accuracy of the result. The scale factor $f$ which ranges from 0.5 to 2 is used to scale the number of nodes which are distributed on the connector. For each type of connector identified by the script, the number of grid points $N$ is defined as the number of nodes for "medium" mesh. The rounded value of $f \times N$ is used as the nodes number for each connector. Thereafter, the advancing-front [13] method is used to

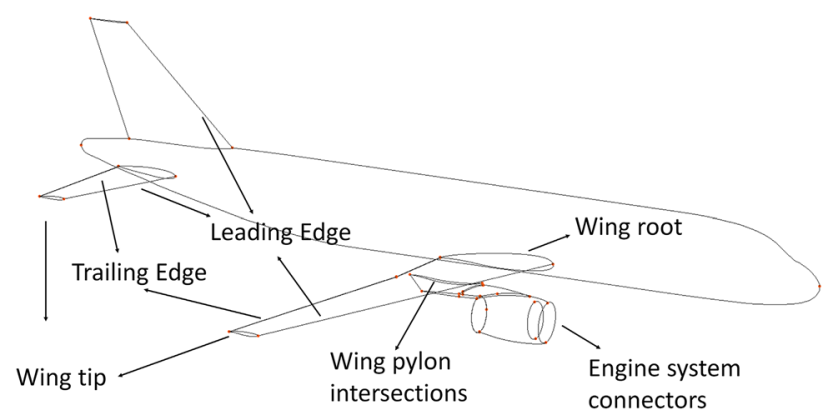

Fig. 2 Connectors to distribute size functions generate surface mesh. For the geometry with high curvature, the boundary is approximated with anisotropic triangle [14] to control the grid size. Then, for the viscous application, anisotropic tetrahedral cells are generated to simulate the boundary layer. The rest of control volume is filled with isotropic tetrahedral cells using Delaunay method [15].

\subsection{CFD Solver}

As soon as the grid is generated with a suitable format for the CFD solver, it is passed to the CFD solver component. Two solvers are used in this study. The open source CFD solver SU2 [16] is chosen for inviscid analysis, and ANSYS Fluent [17] solver is adopted for viscous simulation. SU2 is a finite-volume, cell-based unstructured CFD solver. In this article, the Jameson-Schmidt-Turkel (JST) scheme [18] augmented with artificial dissipation is used for the spatial discretization. In Fluent, a Density-based unstructured solver, cell-based method is chosen. Second order upwind spatial discretization is used to calculate convective fluxes. For viscous term, one-equation SpalartAllmaras (SA) turbulence model is used. All the needed input scripts, and settings for the solvers are generated by the components as an automated process as well.

\section{Verification}

To verify the described process, the implemented chain is initially applied to the well-known test case DLR-F6 wing body configuration. As the reliable and available reference data for this test case are RANS results and wind tunnel data, to verify the surface mesh distribution, RANS simulation are performed.

\subsection{Geometry and computational grids}

To use the CPACS-based automated CFD analysis process, the first step is to assemble a CPACS file. In this study a CPACS file of the DLR-F6 model is assembled by 


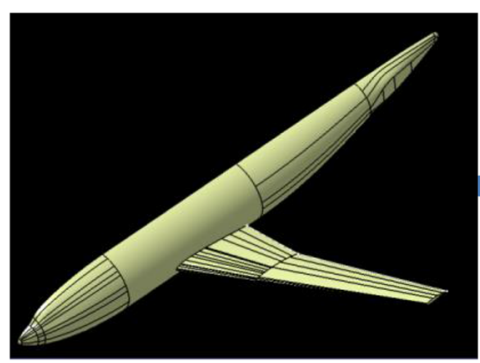

(a) DLR F6 IGES from AIAA DPW

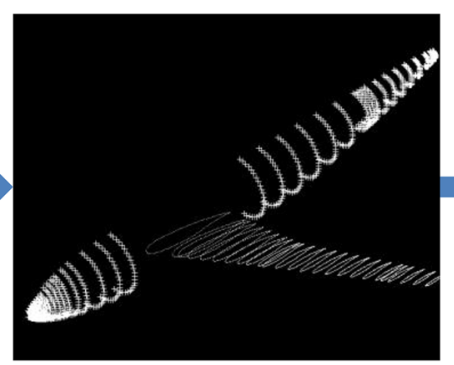

(b) profiles in each slices

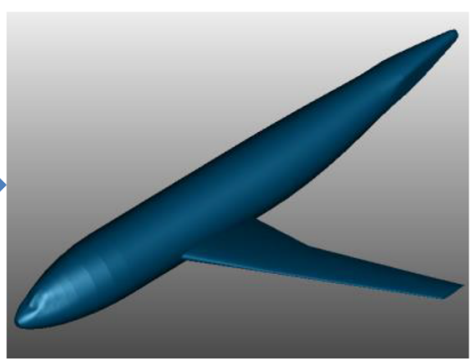

(c) CPACS configuration in TIGL

Fig. 3 CPACS DLR F6 CPACS file initialization

extracting the coordinates of the points from the original DLR-F6 IGES CAD file which comes from 2nd AIAA drag predication workshop (DPW) [19]. The process shows in Fig. 3. The summarized reference data for the DLR-F6 are reported in Table 3 .

As soon as the CPACS file is assembled, the CAD model can be generated with the Geometry generation module depicted in Table 2. Figure 4 shows the comparison of the original CAD file and the one generated from the CPACS file. The gray shade shows the original CAD geometry and the blue shade shows the CAD geometry exported by the Ggeo component. Overall, the two geometries match well with each other. However, at the leading edge of the wing, where the radius is very large, due to the different interpolation methods used for the original CAD file and the OpenCascade-based CAD geometry, some differences can be observed. To investigate the quality of the CAD geometry from the CPACS-TiGL process further, RANS analysis is performed for both of the geometries, and the results are compared with available data. Hybrid grid with approximately 6.5 million of cells is generated for both of the geometries to facilitate the results comparison, and each mesh generation takes around $10 \mathrm{~min}$. The $Y^{+}$value for each grid is approximately 1.0. Figure 5 shows the mesh used in CFD simulations for the TiGL geometry on the geometry surface and symmetry plane.

\subsection{Verification results}

The simulation is performed in fix $C_{\mathrm{L}}$ mode, where $C_{\mathrm{L}}=0.5$ and fix $\mathrm{AOA}$ mode, where $\mathrm{AOA}=0.49^{\circ}$ for each geometry input. All the CFD simulations converge within $8 \mathrm{~h}$ using 4 physical cores. Table 4 reports the AOA, $C_{\mathrm{L}}$ and $C_{\mathrm{D}}$ from each simulation in both modes.

Table 3 Reference quantities for DLR-F6

\begin{tabular}{ll}
\hline$C_{\text {ref }}$ & $141.2 \mathrm{~mm}$ \\
$S_{\text {ref }} / 2$ & $72,700 \mathrm{~mm}^{2}$ \\
$b / 2$ & $585.647 \mathrm{~mm}$ \\
$\mathrm{Ma}$ & 0.75 \\
$R e_{\mathrm{c}}$ & $3 \times 10^{6}$ \\
\hline
\end{tabular}

Wind tunnel experimental data and reference CFD simulation results from NASA [20] are also reported as reference values.

Overall, the flow solver has predicted very close values of $C_{\mathrm{D}}$ for two different input geometries in both modes. In fix $C_{\mathrm{L}}$ model, the difference of drag coefficient between two geometries is within one drag count and both match well with the reference CFD results and the wind tunnel data. The values of the $C_{\mathrm{D}}$ of both geometries are within the $C_{\mathrm{D}}$ range chart of the Drag Prediction Workshop [21]. In fix AOA mode, the $C_{\mathrm{L}}$ and $C_{\mathrm{D}}$ also match very well for both geometry inputs although with small distinction due to difference of the geometry input.

Figure 6 shows the $C_{\mathrm{P}}$ comparison of wind tunnel experimental data against the CFD simulations results for both the input CAD geometries and the reference results at several wing's span-wise stations. The discrepancy between the solution for DPW and the TiGL configuration in both modes is almost invisible except at the leading edge and responding the shock wave location, which indicates the CPACS-TiGL-based CAD file provides a good representation of the original configuration. The results comparison shows the same case with the geometries comparison. Nevertheless, the pressure coefficient at each span-wise section for both the geometries and the reference CFD data shows very good agreement with each other and with the wind tunnel data, which suggests the automated mesh generation process provides a good discretization of the configuration, as well as of the flow field.

\section{CFD application in overall aircraft design}

In this section, the CFD analysis process described in previous sections is used to provide aerodynamics performance within an overall aircraft design task. A short to medium-range transportation aircraft is selected as a test case to demonstrate the impact of the implemented CFD automated chain over the aircraft synthesis process. A grid refinement study is made to determine the resolution accuracy of the mesh used in CFD simulations. The overall 
Fig. 4 Geometry comparison of the TiGL geometry and original CAD file
Fig. 5 Surface and symmetry plane mesh for TiGL configuration
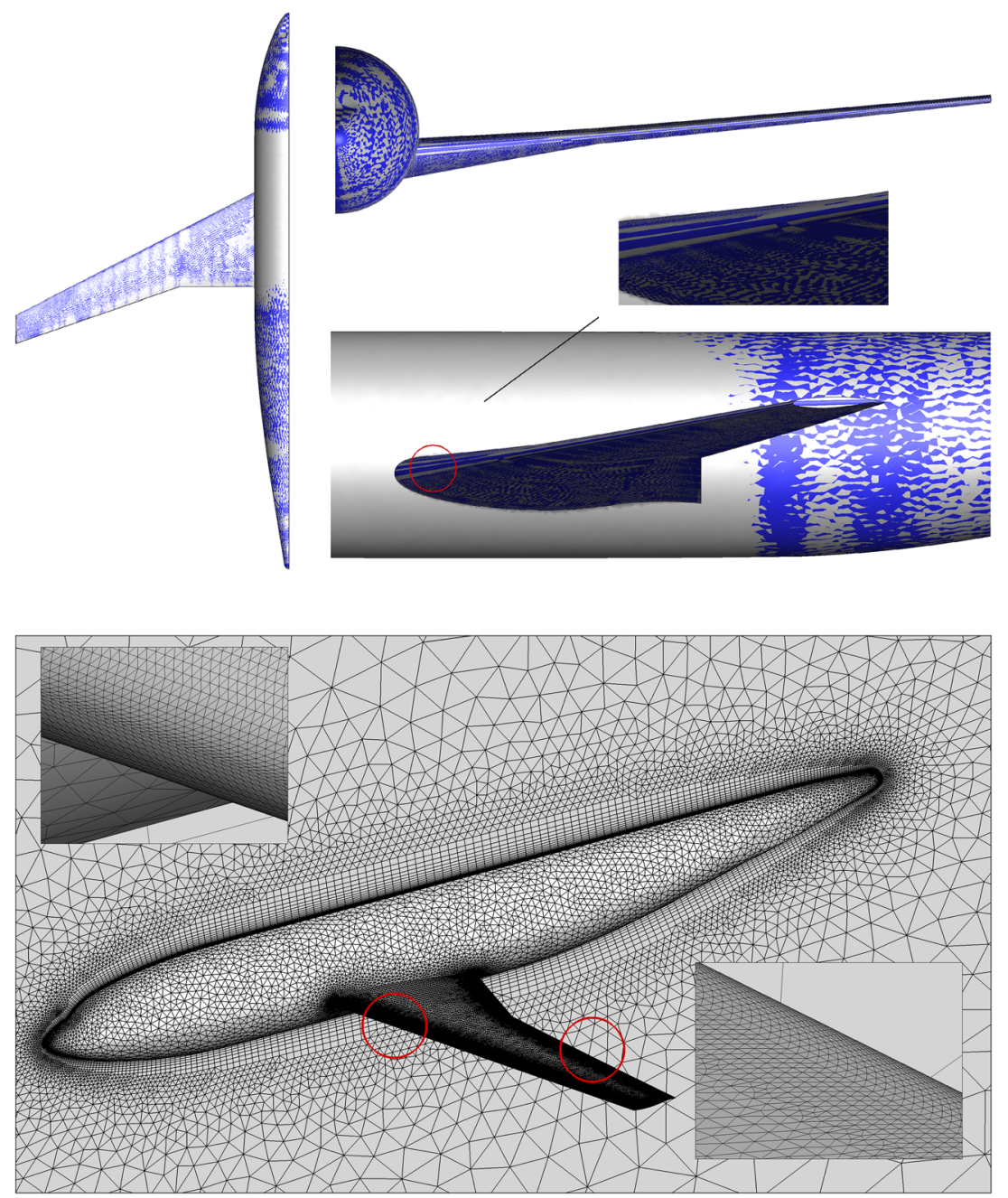

Table 4 Comparison of AOA and $C_{\mathrm{D}}$ at $C_{\mathrm{L}}=0.5$, $\mathrm{Ma}=0.75, R e_{\mathrm{c}}=3 \times \mathrm{e} 10^{6}$

\begin{tabular}{|c|c|c|c|c|c|c|}
\hline \multirow{2}{*}{$\begin{array}{l}\text { DLR_F6 } \\
\text { AOA }\end{array}$} & \multicolumn{2}{|c|}{ DPW_RANS } & \multicolumn{2}{|c|}{ TIGL_RANS } & \multirow{2}{*}{$\begin{array}{l}\text { NASA_USM3D } \\
0.248^{\circ}\end{array}$} & \multirow{2}{*}{$\begin{array}{l}\text { EXP } \\
0.49^{\circ}\end{array}$} \\
\hline & $0.49^{\circ}$ & $0.295^{\circ}$ & $0.49^{\circ}$ & $0.225^{\circ}$ & & \\
\hline$C_{\mathrm{L}}$ & 0.5216 & 0.5000 & 0.5296 & 0.5000 & 0.5 & 0.4984 \\
\hline$C_{\mathrm{D}}$ & 0.02906 & 0.02809 & 0.02932 & 0.02799 & 0.02768 & 0.0293 \\
\hline
\end{tabular}

DPW_RANS and TiGL_RANS are RANS solution for original DPW IGES and TiGL IGES model, respectively

fuel burn obtained by the mission analysis making use of the CFD computed polars is compared with the results obtained by using the VLM solution, and against a pure empirical-based synthesis.

\subsection{Design workflow}

The overall aircraft synthesis process setup in this work is based on a multi-fidelity architecture, to account for the CFD-based analysis process described in the previous sections. The implemented design workflow architecture is shown in Fig. 7.
In the design workflow, the Top Level Aircraft Requirements (TLAR), are specified for the synthesis. The first module is the conceptual aircraft design tool, VAMPzero [22], which is used as aircraft initializer to provide the initial overall synthesis of the aircraft performance, such as the fuel consumption and operating empty mass (OEM). Based on a multi-fidelity architecture, the program allows making use of the aircraft performance values evaluated by external tools. If any of the aircraft characteristics are already defined in the input dataset, they will be directly inherited instead of being recalculated by VAMPzero analysis modules. This feature allows 

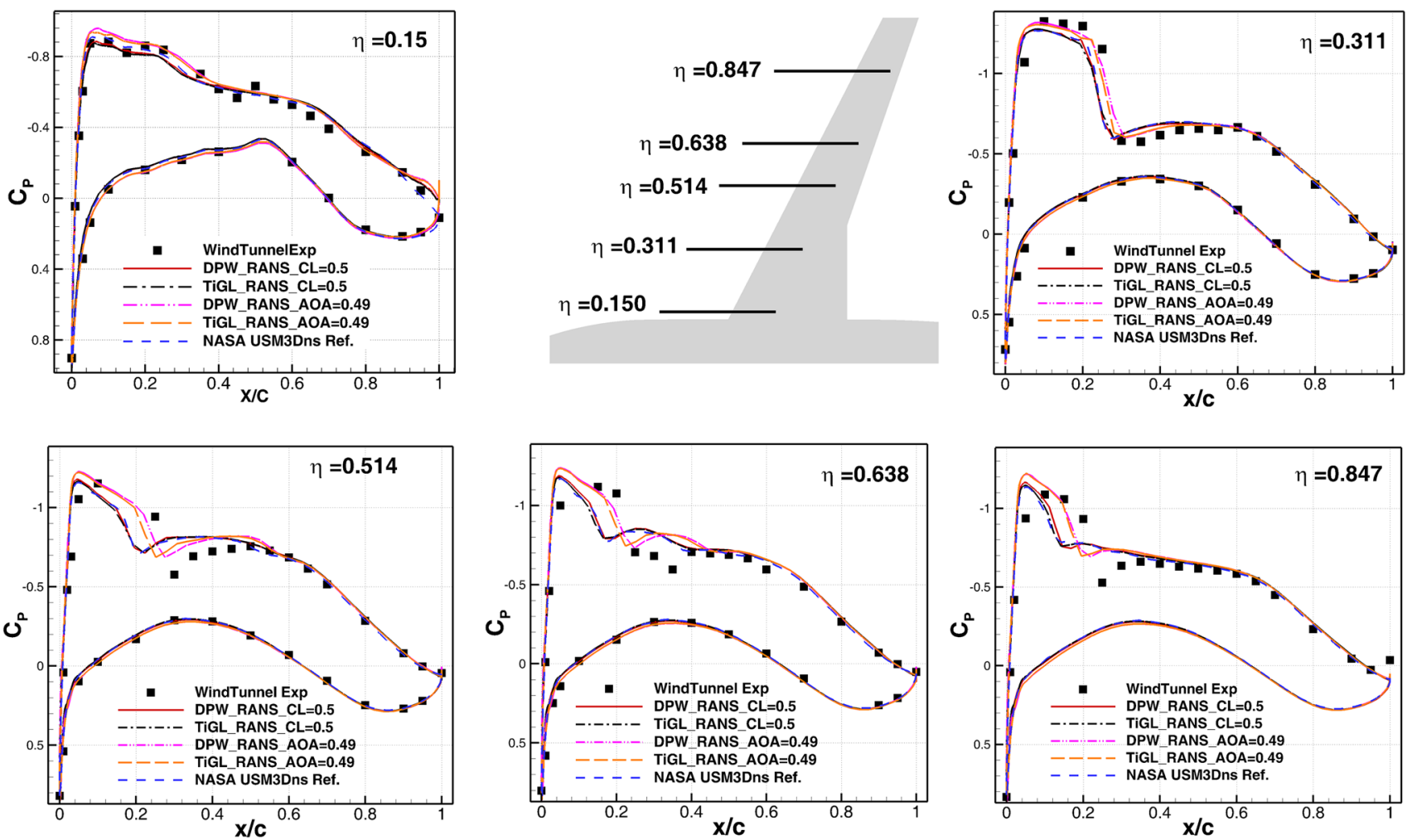

Fig. 6 Comparison of wing surface pressure distributions at $\mathrm{Ma}=0.75$

integrating the presented CFD-based analysis process into the overall synthesis other than using the aerodynamics characteristics estimation available internally to the conceptual tool.

To provide affordable solution, the aerodynamic performance used in this design study is obtained by solving the Euler equations. As a result, the skin friction drag is not accounted into the CFD-based results. To obtain realistic mission fuel values, an estimation of the friction drag is obtained by a method based on the flat plate equivalency for the aircraft wetted area. An available representative engine is chosen to provide performance maps of fuel flow and thrust for pre-defined engines depending on the flight conditions, i.e., Mach number, altitude and thrust setting. Thereafter, mission analysis is performed to simulate an aircraft's flight on a given flight mission profile, and to determine the mission block fuel of the design mission depending on the given aerodynamic polars, the engine performance and the aircraft geometry.

After the configuration is initialized by conceptual design module, the resulting model is progressed to the other analysis components in the workflow. The aerodynamic performances are evaluated with the described CFD analysis chain. The design workflow architecture allows using tools with different levels of fidelity, such as a conceptual tool, VLM method and CFD method, to evaluate the aerodynamic performance for the synthesis process.
Afterwards, the aerodynamics performances are modified by considering friction drag estimation. Hence, mission analysis is performed to update the mission fuel mass based on the conceptual results (e.g., for the design masses), and on the CFD analysis (for the aerodynamics). With the updated mission fuel mass, the design is forwarded once more to the synthesis process, to account for the updates provided by the aerodynamics and mission modules, and to perform an updated synthesis of the aircraft. Currently, in the design process, the geometry of the aircraft at cruise condition is fixed and the aerodynamic performance of the aircraft is assumed to be unchanged. The low speed performance and the control surfaces size of the aircraft are accounted by the conceptual synthesis tool. Thereafter, with the updated synthesized values of OEM and MTOW from the conceptual design tool (VAMPzero in this study), a new mission analysis is performed. The design loop is executed till the convergence of the design masses (OEM, MTOW, and Fuel Mass). In this way, the convergent solution accounts for all the snowball effects in the aircraft synthesis process.

\subsection{Test case}

The configuration used in this design case is the D150, which is an A320 like aircraft and has been used as baseline aircraft in previous studies [23, 24]. The main top level 


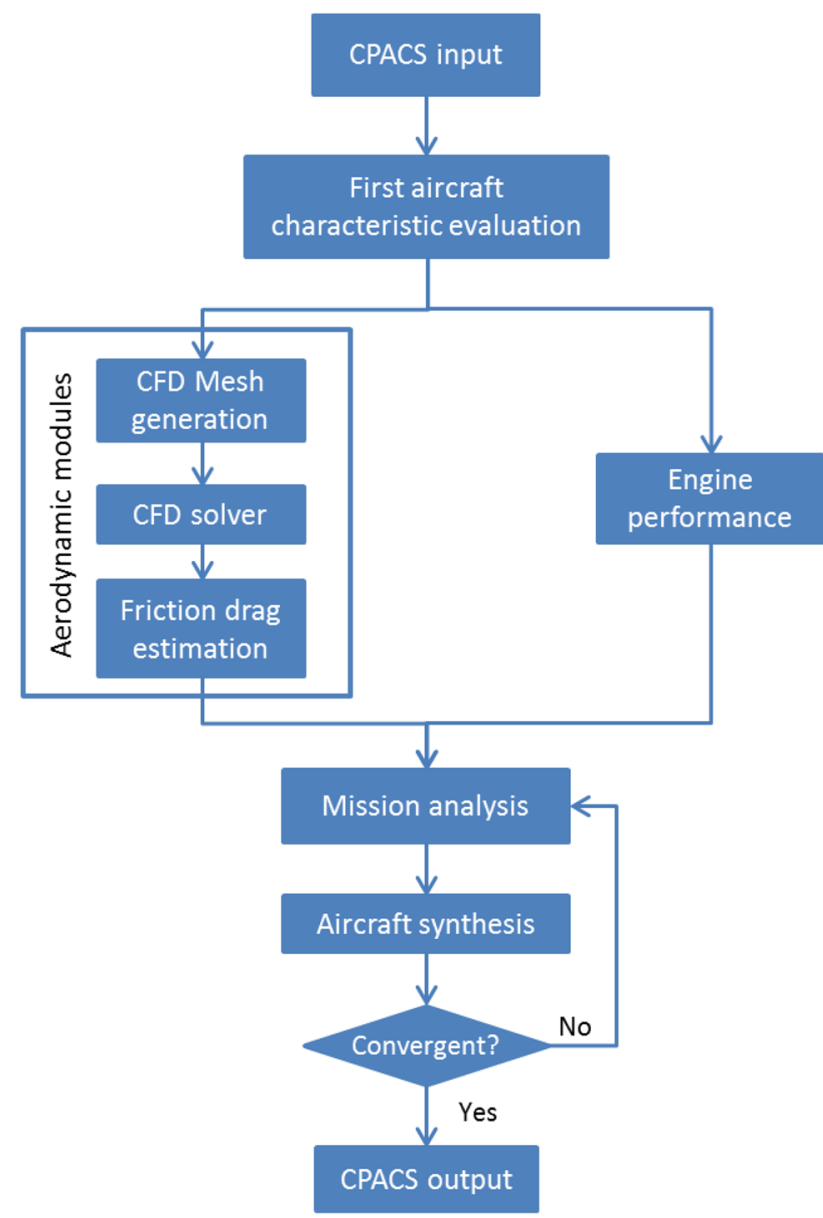

Fig. 7 Workflow of the design process

aircraft requirements (TLAR) are reported in Table 5. Figure 8 shows the initialized configuration, which is in CPACS format, and visualized by the CPACS geometry interpreter TiGL Viewer.

Before the design case is carried out, a grid refinement study is made. A sequence of three refined grids with grid sizes ranging from 0.9 million cells to 2 million cells, named coarse, medium, and fine, respectively, is generated by varying the global factor defined in Ggrid. A series of Angle of Attack (AOA) from $-4^{\circ}$ to $4^{\circ}$ is run for each grid to generate drag polars at the cruise Mach number of $\mathrm{Ma}=0.78$. The polars are shown in Fig. 9. It can be observed that the coarse grid is not sufficiently resolved to match the other two polars. However, the medium and fine grids are nearly indistinguishable from each other.

A description of all grids used in this work, as well as the $C_{\mathrm{D}}$ for each grid at $C_{\mathrm{L}}=0.5$ in $\mathrm{Ma}=0.78$ are given in Table 6 . The medium grid offers $40 \%$ computational savings compared to the fine grid and with acceptable accuracy. To provide an efficient evaluation of the aerodynamic performance, the medium grid is used in the later aircraft synthesis study.
Table 5 TLAR for D150

\begin{tabular}{ll}
\hline Parameter & Value \\
\hline Design range $(\mathrm{km})$ & 4000 \\
PAX & 150 \\
Mach cruise & 0.78 \\
Altitude $(\mathrm{m})$ & 11000 \\
\hline
\end{tabular}

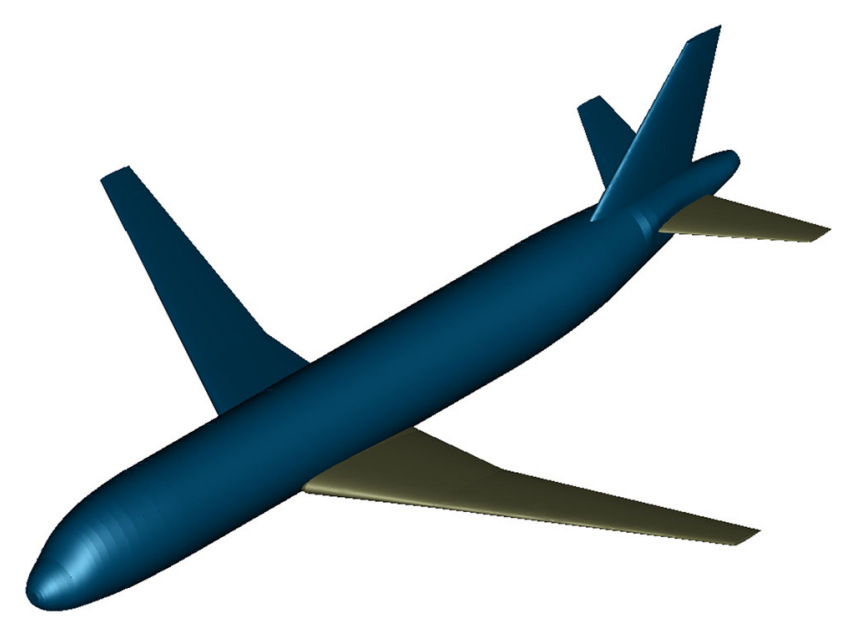

Fig. 8 D150, as visualized in TiGL Viewer

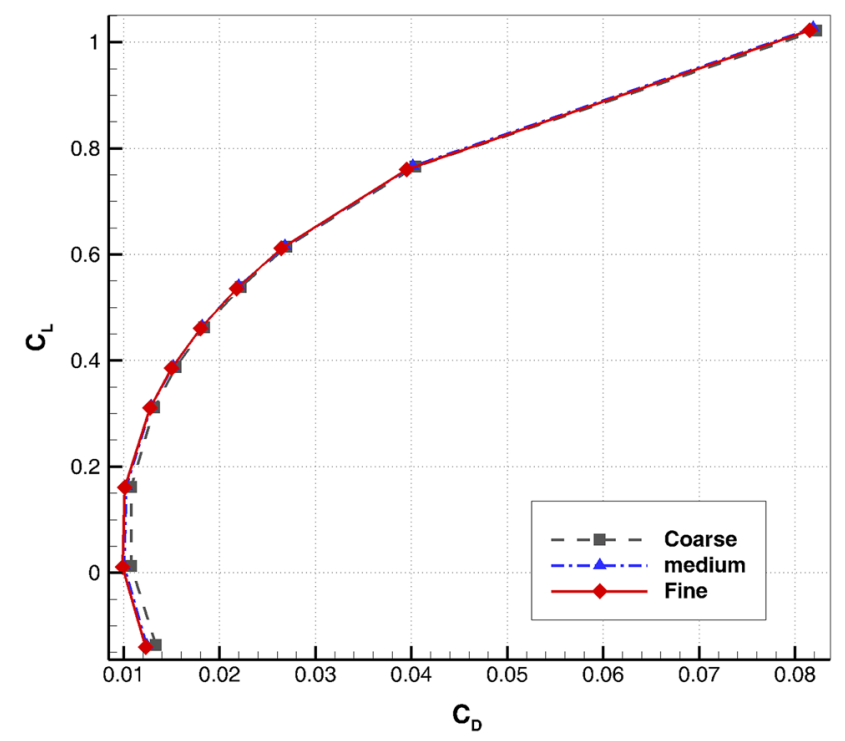

Fig. 9 Drag polars for three levels of refinement

\subsection{Synthesis results}

The overall aircraft synthesis results are compared for three cases:

1. Pure conceptual-based synthesis.

2. Multi-fidelity synthesis with VLM-based aerodynamics. 
Table 6 Mesh sizes and $C_{\mathrm{D}}$ at $C_{\mathrm{L}}=0.5$ in $\mathrm{Ma}=0.78$

\begin{tabular}{lrrl}
\hline Grid & Surface Cells & \multicolumn{1}{l}{ Cells } & \multicolumn{1}{l}{$C_{\mathrm{D}}$} \\
\hline Coarse & 83,905 & 993,604 & 0.02027 \\
Medium & 130,351 & $1,589,162$ & 0.01997 \\
Fine & 169,511 & $2,114,627$ & 0.01996 \\
\hline
\end{tabular}

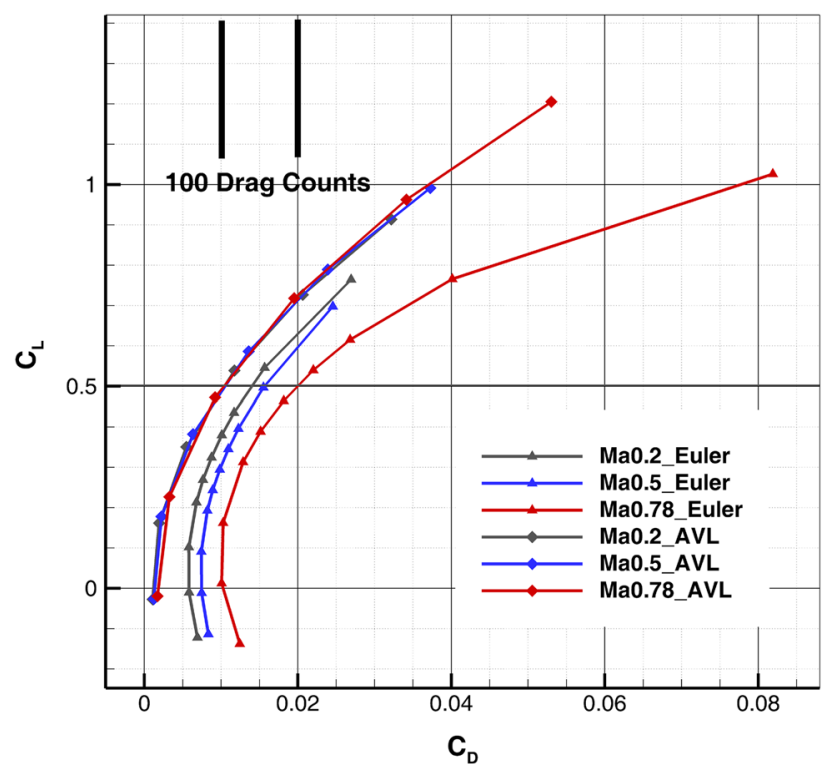

Fig. 10 Comparison of the drag polars with VML and Euler simulation

3. Multi-fidelity synthesis with CFD Euler-based aerodynamics.

Figure 10 shows the comparison of drag polars between VLM method and Euler simulation for Mach number equal to $0.2,0.5$ and 0.78 . An angle of attack sweep from $-4^{\circ}$ to $4^{\circ}$ is run for each grid to generate drag polars at each Mach number. Each polar include 10 points. In total, $30 \mathrm{CFD}$ simulations are performed to estimate aerodynamic behavior of the configuration and each simulation cost 5 min with 4 cores. As expected, the differences between VLM and Euler polars in subsonic regime are relatively small. However, in the cruise condition, due to the high wave drag, significant differences are shown. For example, at $C_{\mathrm{L}}=0.5$, the difference of the $C_{\mathrm{D}}$ can up to $100 \mathrm{drag}$ counts. It worth to be noticed that the wing shape used in CFD analysis is initialized by the conceptual design tool and has not been designed in purpose, which result to unpractical large wave drag compare to that of a satisfactory design where the wave drag takes $4-5 \%$ of the total drag.

The result of the synthesis process, such as the take-off mass (MTOM), fuel mass (MFM) and operating empty mass (OEM) for three synthesis cases are shown in
Table 7 Synthesis results

\begin{tabular}{llcc}
\hline Mass $(\mathrm{Kg})$ & Conceptual & VLM $(\%)$ & Euler CFD $(\%)$ \\
\hline MTOM & 76168 & -2.5 & +5.9 \\
MFM & 13142 & -12.9 & +29.1 \\
OEM & 40527 & -0.1 & +0.4 \\
\hline
\end{tabular}

The results of the VLM and Euler CFD are respect to conceptual values

Table $8 C_{\mathrm{L}}$ and $C_{\mathrm{D}}$ in synthesis cruise condition

\begin{tabular}{llll}
\hline & Conceptual & VLM & Euler CFD \\
\hline$C_{\mathrm{L}}$ & 0.584 & 0.562 & 0.622 \\
$C_{\mathrm{D}}$ & 0.0304 & 0.0271 & 0.0451 \\
\hline
\end{tabular}

Table 7. With extensive available database for conventional configurations, the conceptual synthesis process is calibrated on real aircraft data. As a result, the conceptual design results provide a good reference for comparison. Both multi-fidelity synthesis results, which make use of VLM and Euler CFD simulations, show a difference with the conceptual design case, as shown in Table 7. The main difference for the three cases is due to the fuel consumption at cruise phase, which results from the difference of drag predicted with aerodynamic tools of varying fidelity levels.

Further, due to the snowball effects accounted into the iterative synthesis process, the drag difference results into a different trimming condition at the cruise phase, which is reported in Table 8.

It is obviously that the under estimation of fuel for VLM case is due to the absence of the wave drag. On the other hand, the Euler case tends to give a higher fuel consumption value. The overestimated wave drag at cruise condition results into an increased fuel consumption, and thrust requirements, leading to a higher $C_{\mathrm{L}}$ values to trim the aircraft at the different mission points. Further, as the wave drag predicted by the Euler simulation is highly sensitive to the wing's geometrical representation, it is crucial to provide suitable input file to the CFD-based synthesis process. Hence, the transition from the conceptual to the CFD-based analysis, needs to account for an enhancement of the geometry quality as well. The automated process presented here may be further extended to generate proper wing shape for CFD simulation within the multi-fidelity synthesis at the early design stages, and this work will also help to decrease redesign effort at the later stages.

\section{Conclusions and outlook}

In this paper, an automated CFD analysis chain is implemented. The objective is to improve the prediction of the aerodynamic behavior for conventional and unconventional 
aircraft configurations in the pre-design stages, and to include automated CFD-based analysis into the overall aircraft synthesis process. The chain is verified with the well-known test case DLR-F6. The results show the implemented process provides a high quality representation of geometry and a good representation of the aerodynamic performance.

With the centralized CPACS data modeling, a multifidelity aircraft synthesis process is implemented by making use of automated CFD-based analysis process deployed in RCE framework. The design synthesis is performed with different levels of fidelity. As expected, by taking wave drag into account, the synthesis results with Euler simulation shows higher fuel consumption compare with VLM results. Further, by giving an overestimated drag, the CFD simulation results into much higher mission fuel consumption compared with the purely conceptual design method. Source of the drag overestimation is also due to the representation of the wing design, which is initialized by the conceptual synthesis (as expected). Hence, this study highlights the complexities faced by the designer when introducing physics-based analysis in the predesign stage, and the necessity to provide suitable geometries for the analysis modules.

Nevertheless, to have a better understanding on the aircraft characteristics, it is of great meaningful to introduce CFD-based analysis into the overall aircraft design, especially for unconventional aircraft configuration, where the flow physics requires deeper investigations, and when strong interactions between different disciplines will occur, for example between aerodynamics and propulsion. Further, introducing automated CFD-based analysis into the early aircraft synthesis, is expected to minimize the redesign activities at the later stages.

In the following studies, it is expected to use aerodynamic shape design to initialize a suitable aircraft configuration for OAD making use of CFD analysis and optimization techniques. Effective methods are reported in this field, which make use of gradient-based optimization algorithm in conjunction with an adjoint method for the computation of the required shape derivatives.

Acknowledgements The research presented in this paper has been performed in the framework of the AGILE project (Aircraft 3rd Generation MDO for Innovative Collaboration of Heterogeneous Teams of Experts) and has received funding from the European Union Horizon 2020 Programme (H2020-MG-2014-2015) under grant agreement no 636202. The authors are grateful to the partners of the AGILE Consortium for their contribution and feedback.

Open Access This article is distributed under the terms of the Creative Commons Attribution 4.0 International License (http://crea tivecommons.org/licenses/by/4.0/), which permits unrestricted use, distribution, and reproduction in any medium, provided you give appropriate credit to the original author(s) and the source, provide a link to the Creative Commons license, and indicate if changes were made.

\section{References}

1. CAO.: Aviation and Climate Change. International Civil Aviation Organization (ICAO) Environmental Report, (2010)

2. http://www.acare4europe.com/sria(2016). Accessed 20 July 2016

3. Liebeck, R.H.: Design of the blended wing body subsonic transport. J. Aircr. 41, 10-25 (2004)

4. Gundlach, J.F., Philippe-André, Té, et al.: Conceptual design studies of a strut-braced wing transonic transport. J. Aircr. 37(6), 976-983 (2000)

5. Rizzi, A.: Modeling and simulating aircraft stability and control-the SimSAC project. Prog. Aerosp. Sci. 47(8), 573-588 (2011)

6. Ciampa, P.D., Zill, T., Nagel, B.: A hierarchical aeroelastic engine for the preliminary design and optimization of the flexible aircraft. In: 54th AIAA/ASME/ASCE/AHS/ASC Structures, Structural Dynamics, and Materials Conference, Boston, Massachusetts, USA (2013)

7. Ciampa, P.D., Nagel, B., La R.: Gianfranco Preliminary Design for Flexible Aircraft in a Collaborative Environment. In: 4th CEAS Air and Space Conference, Linköping, Sweden (2013)

8. http://www.cpacs.de/ (2016). Accessed 20 July 2016

9. Bachmann, A., Kunde, M., Litz, M., et al.: Advances in generalization and decoupling of software parts in a scientific simulation workflow system. In: The Fourth International Conference on Advanced Engineering Computing and Applications in Sciences Florence, Italy (2010)

10. http://www.opencascade.com/ (2016). Accessed 20 July 2016

11. http://rcenvironment.de/ (2016). Accessed 20 July 2016

12. http://www.pointwise.com (2016). Accessed 20 July 2016

13. Löhner, Rainald, Parikh, Paresh: Generation of three-dimensional unstructured grids by the advancing-front method. Int. J. Numer. Meth. Fluids 8(10), 1135-1149 (1988)

14. Pirzadeh, S.: Unstructured viscous grid generation by the advancing-layers method. AIAA J. 32(8), 1735-1737 (1994)

15. Watson, D.F.: Computing the n-dimensional delaunay tessellation with application to voronoi polytopes. Comput. J. 24(2), 167-172 (1981)

16. Palacios, F., Economon, T.D., Aranake, A., Copeland, S.R., Lonkar, A.K., Lukaczyk, T.W., Manosalvas, D.E., Naik, K.R., Padron, S., Tracey, B., Variyar, A., Alonso, J.J.: Stanford University Unstructured (SU2): Analysis and Design Technology for Turbulent Flows, in 52nd Aerospace Sciences Meeting. National Harbor, Maryland (2014)

17. http://www.ansys.com/Products/Simulation+Technology/Fluid+ Dynamics/Fluid+Dynamics+Products/ANSYS+Fluent (2016). Accessed 20 July 2016

18. Jameson, A., Schmidt, W., Turkel, E.: Numerical solutions of the Euler equations by finite volume methods using Runge-Kutta time-stepping schemes. AIAA Paper 1259, 1981 (1981)

19. http://aaac.larc.nasa.gov/tsab/cfdlarc/aiaa-dpw/Workshop2 (2016). Accessed 20 July 2016

20. Lee-Rausch, E.M., Frink, N.T., Mavriplis, D.J., et al.: Transonic drag prediction on a DLR-F6 transport configuration using unstructured grid solvers. Comput. Fluids 38(3), 511-532 (2009)

21. http://aiaa-dpw.larc.nasa.gov/Workshop2/pdf/81Laflin_DPW2Da taSumry.pdf (2016). Accessed 20 July 2016

22. Bohnke, D., Nagel, B., Gollnick, V.: An approach to multi-fidelity in conceptual aircraft design in distributed design environments. In Proceedings of the 2011 IEEE Aerospace Conference (pp. 1-10). IEEE Computer Society. Big Sky, USA (2011) 
23. Ciampa, P.D., Zill, T., Nagel, B.: Aeroelastic Design and Optimization of Unconventional Aircraft Configurations in a Distributed Design Environment, AIAA-2012-1925, 53rd AIAA/ ASME/ACSE Structures, Structural Dynamics and Materials Conference, Hawaii, (2012)
24. Zill, T., Ciampa, P.D., Nagel, B.: A Collaborative MDO Approach for the Flexible Aircraft,54th AIAA/ASME/ASCE/ AHS/ASC Structures, Structural Dynamics, and Materials Conference, Boston, (2013) 